\title{
Changes of Upper Gastrointestinal Symptoms and Endoscopic Findings in Japan Over 25 Years
}

\author{
Noriaki Manabe ${ }^{1}$, Ken Haruma ${ }^{2}$, Tomoari Kamada ${ }^{2}$, Hiroaki Kusunoki ${ }^{3}$, Kazuhiko Inoue ${ }^{3}$, \\ Takahisa Murao ${ }^{2}$, Hiroshi Imamura ${ }^{1}$, Hiroshi Matsumoto ${ }^{2}$, Ken-ichi Tarumi ${ }^{2}$, \\ Akiko Shiotani $^{2}$ and Jiro Hata ${ }^{1}$
}

\begin{abstract}
Background and Aims In Japan, the prevalence of Helicobacter pylori infection has decreased recently, but there has been little longitudinal research on the changes of symptoms and endoscopic findings over many years. The aim of this study was to evaluate the changes in endoscopic findings and symptoms over time in patients undergoing upper gastrointestinal endoscopy for assessment of upper abdominal symptoms.

Methods Over a period of 25 years, between 1981 and 2005, we performed three investigations (1981-2, 1990-1 and 2004-5) on the symptoms and endoscopic findings of patients undergoing endoscopy at Kawasaki Medical School Hospital for the assessment of upper abdominal symptoms.

Results The most common complaint for all ages was "discomfort and/or pain". Over time, the percentage of patients complaining of "heartburn" increased almost 5-fold. With respect to endoscopic findings, the prevalence of peptic ulcers decreased, whereas the detection of no abnormalities and the detection of erosive esophagitis increased.

Conclusion In the future in Japan, there will be fewer patients with peptic ulcers and more patients with gastro-esophageal reflux disease or dyspeptic patients who display no endoscopic abnormal findings.
\end{abstract}

Key words: gastrointestinal symptom, dyspepsia, heartburn, endoscopy, gastro-esophageal reflux disease, peptic ulcer

(Intern Med 50: 1357-1363, 2011)

(DOI: 10.2169/internalmedicine.50.4731)

\section{Introduction}

There have been considerable changes in the epidemiology of upper gastrointestinal (GI) disease in the Asia-Pacific region over the past decade (1). A decline in the prevalence of peptic ulcer disease in the West over the past three to four decades has been reported (2). Recently, similar observations have been made in the Asia-Pacific region as well (3). At the same time, gastro-esophageal reflux disease (GERD) has been reported to be increasing in the AsiaPacific region as well as in Western countries $(4,5)$. To observe these changes is important, because such observation of the changing epidemiology of a disease may provide valuable insight as to the possible pathogenic mechanisms.

Upper GI endoscopy is an accurate and safe method of evaluating the mucosa of the esophagus, stomach and duodenum, and it is performed for a variety of indications. Endoscopy is the first diagnostic procedure undertaken to evaluate patients presenting acute or chronic dyspeptic symptoms. Dyspepsia is now defined as a chronic or frequently recurring upper GI tract condition with symptoms such as epigastric pain or discomfort, and is believed to originate in the gastroduodenal region (6). It is a frequent reason for attending primary care consultations and undergoing an endoscopic examination (7), and it has a great impact

\footnotetext{
${ }^{1}$ Division of Endoscopy and Ultrasonography, Department of Clinical Pathology and Laboratory Medicine, Kawasaki Medical School, Japan, ${ }^{2}$ Division of Gastroenterology, Department of Internal Medicine, Kawasaki Medical School, Japan and ${ }^{3}$ Department of Health Care Medicine, Kawasaki Medical School, Japan

Received for publication October 23, 2010; Accepted for publication March 14, 2011

Correspondence to Dr. Noriaki Manabe, n_manabe@hkg.odn.ne.jp
} 
Table 1. Characteristics of the 3 Groups

\begin{tabular}{llll}
\hline Period & $1981-1982$ & $1990-1991$ & $2004-2005$ \\
\hline Number of patients & 352 & 914 & 1724 \\
Gender (M/F) & $230 / 122$ & $590 / 324$ & $1014 / 710$ \\
Mean age (years) & $49.5 \pm 16.7$ & $52.2 \pm 16.4$ & $59.6 \pm 17.0$ \\
\hline
\end{tabular}

M, male; F, female

on the patient's quality of life (8). Although recently complaints of heartburn have been reported to be increasing in the Asia-Pasific region $(4,5)$, there have no reports in Japan regarding the long-term changes in upper GI symptoms with indications for upper GI endoscopy, such as dyspepsia. Accordingly, the aim of this study was to assess changes in endoscopic findings and symptoms over the past 25 years in Japan.

Abbreviations: GERD: gastro-esophageal reflux disease, GI: gastrointestinal

\section{Patients and Methods}

The medical records of patients who underwent an upper GI endoscopy in the years from 1981 to 1982,1990 to 1991 and 2004 to 2005 in our hospital were retrospectively evaluated. Our hospital is the major endoscopic center in our area. Data for this study were retrieved from a computerized endosopy database. All endoscopic procedures were performed by one of 11 experienced endoscopists or by a resident in gastroenterology under the supervision of an experienced endoscopist. This study was approved by the hospital ethics committee and authorization for the use of the medical records for research purposes was confirmed before they were accessed.

The indications for the upper GI endoscopies and the endoscopic findings in the patients were recorded. Indications for the upper GI endoscopies were classified into the following categories of symptoms: epigastric pain or discomfort, heartburn, nausea, dysphagia, hematemesis/melena and others. In this study, a dyspeptic symptom was defined as symptoms of "discomfort and/or pain". If patients had two or more symptoms, the most prominent symptom was selected as the indication for endoscopy.

Endoscopic findings were classified into the following disease categories: normal findings, erosive esophagitis (EE), advanced gastric carcinoma (GCA), gastric ulcer (GU), duodenal ulcer (DU) and others. A diagnosis of normal was made when no focal lesions were observed, so endoscopic detection of gastritis without any focal lesions was classified as normal. GU or DU scars were also not included as focal lesions. However, acute gastric mucosal lesions, acute gastroduodenal lesions and nodular gastritis were not classified as normal. A diagnosis of EE was made when mucosal breaks were observed. When patients had two or more focal lesions, we classified each lesion separately. That is, if a patient had both a GU and a DU, we counted the two ul- cers separately. All patients older than 18 years old were assessed, except for those who had undergone a scheduled therapeutic endoscopy and those with previous endoscopic evaluations for the same reason.

\section{Statistical analysis}

Results are expressed as the mean \pm standard deviation. Analysis of variance was used to compare the mean age of the patients in each study period. The $\chi^{2}$ test, with Yates' correction if required, was used to compare proportional data (the symptoms that were indications for upper GI endoscopy and the endoscopic findings) over the study period. A $\mathrm{p}$ value of less than 0.05 was considered to indicate significance.

Abbreviations: EE: erosive esophagitis, GCA: advanced gastric carcinoma, GU: gastric ulcer, DU: duodenal ulcer

\section{Results}

\section{Patient characteristics}

A total of 3,008 patient files were reviewed for this study. The mean age of the patients was $56.2 \pm 17.1$ years. There were 352 patients from 1981 to 1982 , 914 patients from 1990 to 1991 and 1,724 patients from 2004 to 2005. We observed an almost 5-fold increase in the number of endoscopies performed over the 25 -year period. The mean age of the patients from 2004 to 2005 tended to be older than those from 1981 to 1982 . The number of male patients was 1.8fold larger than that of female patients in 1981-1982 and 1.4-fold larger in 2004-2005. Thus the gender differences in indication trends for upper GI endoscopy were becoming smaller (Table 1).

\section{Trends of indications for upper gastrointestinal en- doscopy}

The most common indication was "discomfort and/or pain" throughout the study period. We observed an almost 5 -fold increase in the percentage of patients with the symptom of "heartburn" over the 25 -year period (from $1.7 \%$ in $1981-1982$ to $8.2 \%$ in $2004-2005, \mathrm{p}<0.05)$. On the other hand, hematemesis and melena gradually became less frequent (from $23.6 \%$ in $1981-1982$ to $15.2 \%$ in $2004-2005$, p $<0.05$ ) (Fig. 1). 


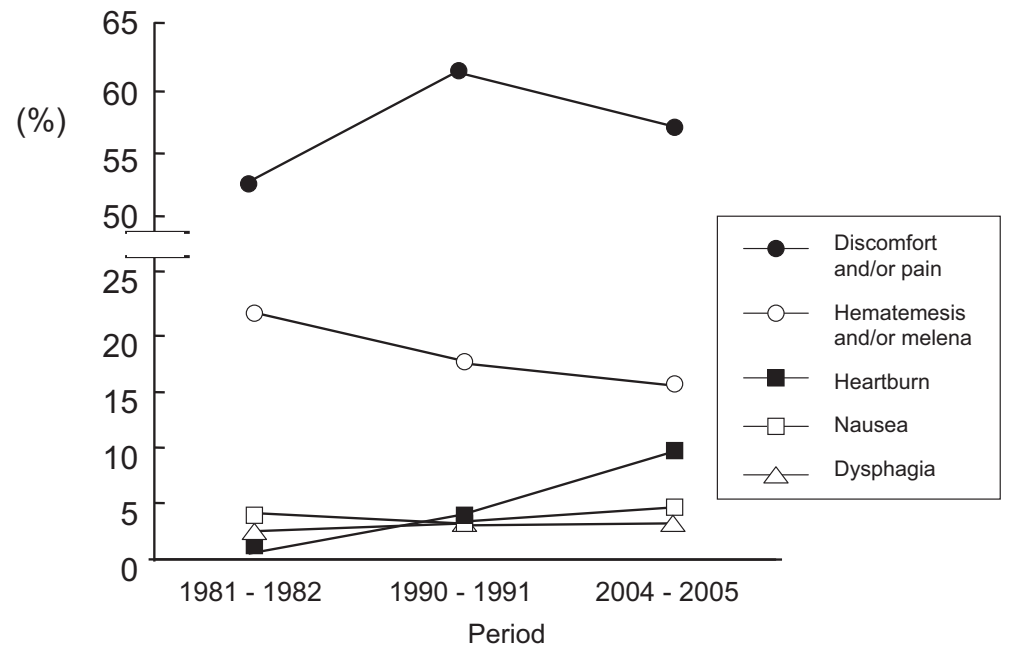

Figure 1. Trends in the indications for upper GI endoscopy during the 25-year study period. The most frequent indication was "discomfort and/or pain". "Heartburn" showed an increase, while "hematemesis or melena" showed a decrease.

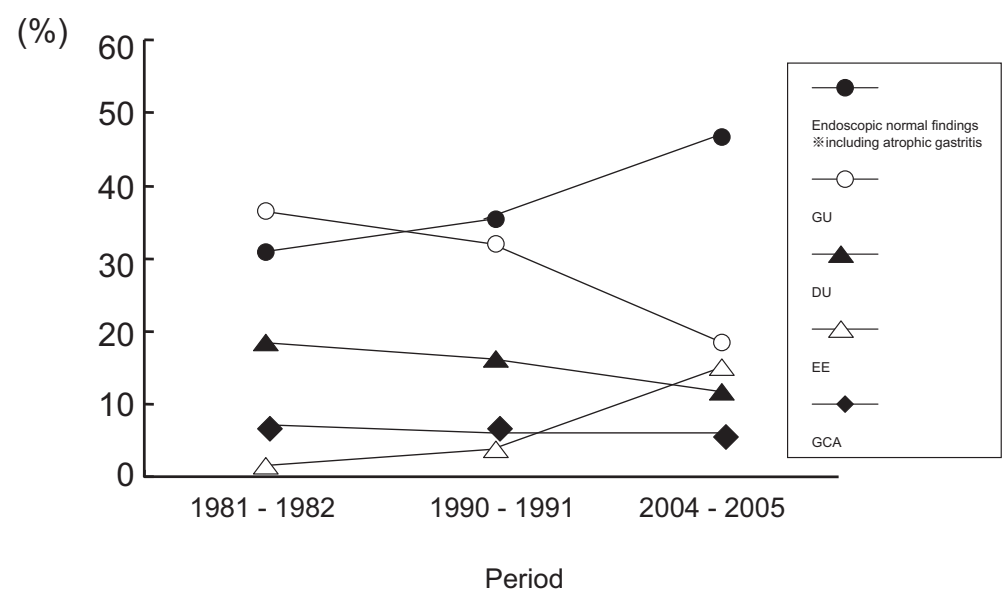

Figure 2. Trends of upper GI endoscopic findings during the 25-year study period. GU: gastric ulcer, DU: duodenal ulcer, EE: erosive esophagitis, GCA: advanced gastric carcinoma. Both normal findings and EE increased, whereas GU decreased. The prevalence of GCA remained low over the entire 25 years.

\section{Trends of upper gastrointestinal endoscopic find- ings}

The percentage of patients with normal endoscopic findings increased significantly over the 25 -year period $(31.0 \%$ in $1981-1982,35.7 \%$ in $1990-1991$ and $48.2 \%$ in $2004-$ $2005, \mathrm{p}<0.05$ comparison between 1981-1982 and 20042005). The percentage of patients with EE also increased significantly over the three periods $(2.0 \%$ in $1981-1982$, $4.2 \%$ in $1990-1991$ and $14.3 \%$ in $2004-2005$, p $<0.05$ comparison between 1981-1982 and 2004-2005). However, GU showed a significant decrease over the total period of study (38.1\% in $1981-1982,31.6 \%$ in $1990-1991$ and $19.7 \%$ in 2004-2005, p<0.05 comparison between 1981-1982 and 2004-2005). DU also tended to decrease, but there was no significant change. On the other hand, the prevalence of GCA remained low throughout the three periods $(7.4 \%$ in
$1981-1982,7.3 \%$ in $1990-1991$ and $4.0 \%$ in $2004-2005)$ (Fig. 2).

We performed further analysis of the two main symptoms. First, we investigated the trends of endoscopic findings over the study period in patients complaining of "discomfort and/ or pain", that is to say "dyspeptic symptoms" (Fig. 3). The percentage of dyspeptic patients with normal endoscopic findings increased $(42.0 \%$ in $1981-1982,45.6 \%$ in 1990 1991 and $58.4 \%$ in $2004-2005, \mathrm{p}<0.05$ comparison between 1981-1982 and 2004-2005), whereas those with peptic ulcers declined over the 25-year period $(52.0 \%$ in 1981-1982, $46.2 \%$ in $1990-1991$ and $24.8 \%$ in $2004-2005$, p $<0.05$ comparison between 1981-1982 and 2004-2005).

Next, we investigated the trends of endoscopic findings over the study period in patients complaining of "heartburn" (Fig. 4). We found that the percentage of patients with EE among patients complaining of "heartburn" showed a sig- 


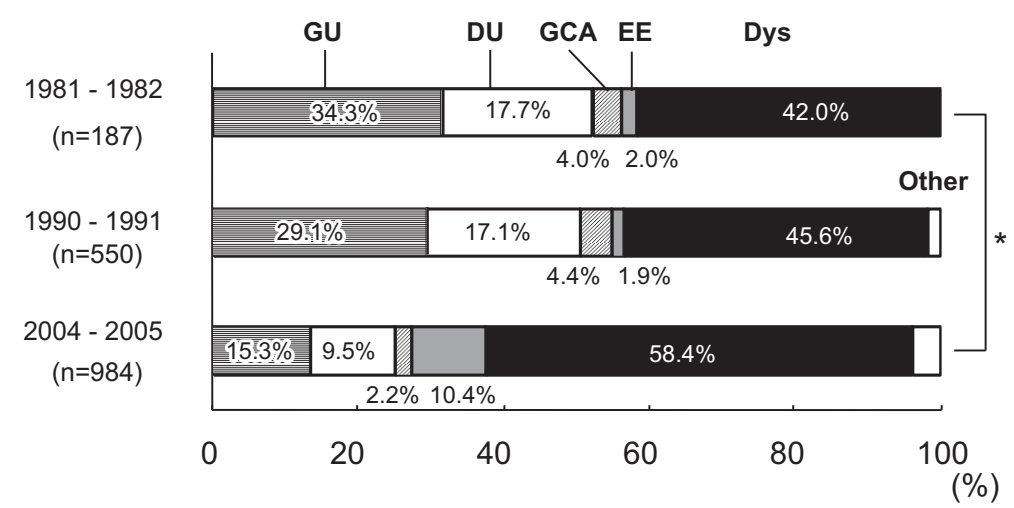

Figure 3. Trends of upper GI endoscopic findings in patients complaining of "discomfort and/or pain”. GU: gastric ulcer, DU: duodenal ulcer, GCA: advanced gastric carcinoma, EE: erosive esophagitis, Dys: dyspeptic patients with normal endoscopic findings. ${ }^{*} \mathbf{p}<0.05$. The percentage of patients with GU, DU, and GCA decreased, while the percentage of patients with EE or Dys increased significantly.

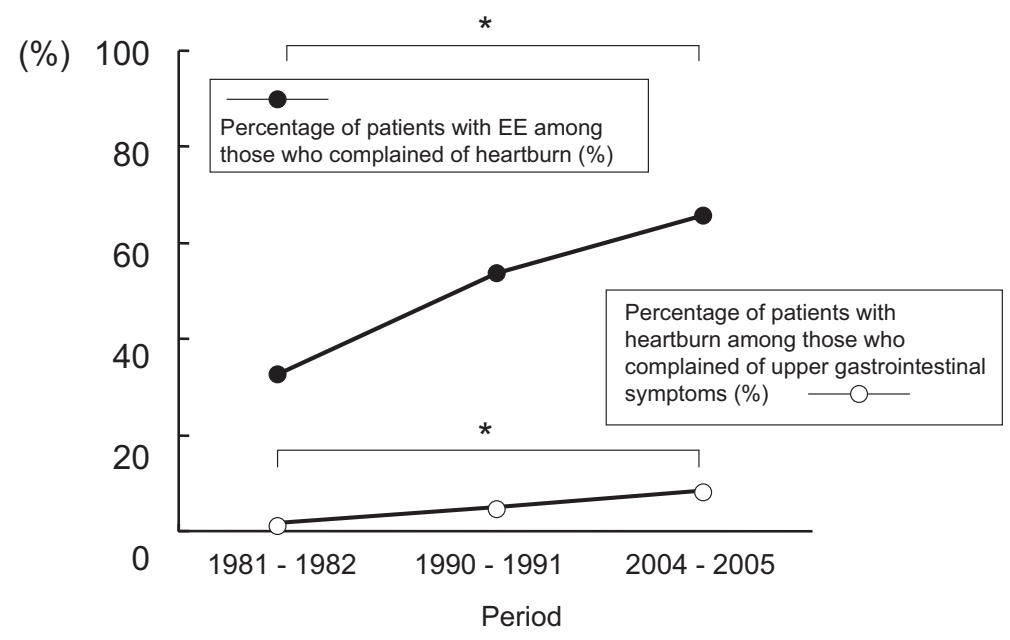

Figure 4. Trends of EE among patients with heartburn symptoms. EE, erosive oesophagitis. $* p<0.05$. The percentage of patients with EE increased markedly among those with heartburn symptoms.

nificant increase (35.2\% in 1981-1982, 55.3\% in 1990-1991 and $62.4 \%$ in $2004-2005, \mathrm{p}<0.05$ comparison between 1981 1982 and 2004-2005).

\section{Discussion}

The present study revealed changes of the symptomatic indications for upper GI endoscopy and endoscopic findings over a 25-year period in Japan. "Discomfort and/or pain" was the most common upper GI symptom leading to endoscopy over the entire 25-year period, but the endoscopic diagnoses changed greatly. The percentage of patients with focal endoscopic lesions decreased, whereas dyspeptic patients with normal endoscopic findings or GERD patients showed an increase.

In this study, we found that the number of male patients was 1.8-fold larger than that of female patients in 19811982 and 1.4-fold larger in 2004-2005. With regard to the gender differences in each upper GI disease, peptic ulcer diseases were predominant in male patients, whereas there were no significant gender differences in dyspeptic patients who display no endoscopic abnormal findings. That is, the gender differences in indication trends for upper GI endoscopy became smaller according to the decreasing number of peptic ulcer diseases and the increasing number of dyspeptic patients who display no endoscopic abnormal findings.

This study revealed that the most common indication for endoscopy was "discomfort and/or pain" throughout the study period. Dyspepsia is a common disorder with a reported prevalence ranging from $13 \%$ to $40 \%$ in Western countries (9). Approximately $60 \%$ of patients with dyspeptic symptoms had functional dyspepsia (FD) in previous studies, meaning that no organic cause of their upper GI tract symptoms could be found $(10,11)$. In this study, the prevalence of patients with normal endoscopic findings was $58.4 \%$ (575/984) among dyspeptic patients, which was consistent with previous reports $(10,11)$. However, one ques- 
tion that may arise is whether the incidence of normal endoscopic findings shown in this study is different from the actual incidence. Empirical antisecretory therapy has long been tried as an initial management option for dyspeptic patients. In a previous Danish randomized trial, patients suffering from dyspepsia showed no difference in symptoms one year after their initial endoscopy or acid suppression therapy, but early endoscopy led to greater patient satisfaction and reduced the number of consultations and prescriptions (12). In Japan, the cost of endoscopy is relatively cheap, so endoscopists prefer to assess the upper GI tract as part of the initial management of patients with dyspepsia. Therefore, it seems likely that the incidence of normal endoscopic findings shown in this study was not markedly different from the actual incidence.

The current study revealed that the percentage of dyspeptic patients with normal endoscopic findings increased over the last 25 years. It is presumed that there will be more dyspeptic patients in the future, and it is not realistic in daily practice to perform upper GI endoscopy for all dyspeptic patients. However, the best screening method for discriminating these dyspepsia from organic disease has yet to be established $(13,14)$. The use of alarm symptoms alone to select dyspeptic patients for endoscopy, in order to avoid a large number of negative endoscopies, may lead to overlooking a small number of patients with curable esophagogastric cancers (15). A strategy for the selection of endoscopy candidates that employs a predictive model based on $H$. pylori status, plus clinical characteristics and symptoms, would be more cost effective $(12,16)$. In the present study, despite the increased rate of upper GI endoscopies over the 25-year period, which was mainly for the evaluation of patients with dyspeptic symptoms, we did not achieve an improvement in the endoscopic detection of GCA and the detection rate of GCA did not increase during the observation period. On the other hand, the percentage of patients with normal endoscopic findings showed a rapid increase over the years. In addition, endoscopic findings rarely alter therapy, since most patients with dyspeptic symptoms receive acid-suppressive treatment after endoscopy anyway $(17,18)$. Therefore, we suggest that patients who have dyspepsia without alarm symptoms, clinical characteristics and $H$. pylori infection should not initially undergo an upper GI endoscopy. A trial of proton pump inhibitor therapy for 1 month might be a more justifiable initial approach, even in Japan, where the cost of endoscopy is relatively cheap.

Among several indications, we observed a 5-fold increase in patients with "heartburn" over the 25 years of the study and many of them had associated EE. At present, GERD appears to be one of the common chronic GI tract disorders in Japan as well as in Western countries (19). This trend is thought to be linked to a decrease of $H$. pylori and an increase of GERD. Although this study did not evaluate changes in $H$. pylori infection, there have been many reports regarding the decrease of $H$. pylori infection rates in Japan $(20,21)$. Furthermore, a plausible mechanism for
GERD is the creation of a "healthier" stomach with higher acid production, which is a consequence of eliminating $H$. pylori infection (22). We also speculated that the increasing prevalence of esophageal hiatus hernia is related to the increasing prevalence of GERD patients, although we did not investigate the time trend in the prevalence of esophageal hiatus hernia. Esophageal hiatus hernia has already been shown to play an important role in the pathogenesis of EE complaints of "heartburn" (23). Furthermore, several recent several studies have demonstrated that the prevalence of esophageal hiatus hernia is increasing in East Asia, as well as in Western countries $(24,25)$. Therefore, we speculated that the increasing prevalence of esophageal hiatus hernia during the study period of over 25 years is one of the factors related to the increasing prevalence of GERD patients. Other environmental factors loosely classified as lifestyle changes, which include changes in diet, smoking and drinking, as well as increasing obesity, also likely play an important role in the observed increase of $\operatorname{EE}(26,27)$ - even allowing for the increasing diagnosis of EE through better awareness of the disease amongst doctors and endoscopists.

The present study also showed that peptic ulcers had decreased in incidence over the last 25 years. A similar observation has been reported in several other Asian studies $(28,29)$. In the first period of the present study, we noted that the rate of GU was almost 2-fold higher compared with DU-consistent with the results of previous studies in Japan $(30,31)$. However, this changed over the 25year period as the dominance of GU over DU decreased, with the prevalence of GU and DU becoming comparable in recent times, consistent with the report of Goh et al (32). A possible explanation for this change is the decreased prevalence of $H$. pylori infection.

This study also showed that changes in the incidence of GCA over time have been more gradual than those of peptic ulcer disease. The reason for this may be that the pathology of GCA takes a longer time to evolve. H. pylori should have an important role in the development of GCA, but a change in the prevalence of GCA cannot be expected solely on the basis of $H$. pylori eradication. In Western countries, the incidence of GCA started to decline even before the discovery of $H$. pylori in 1982 . We speculate that socioeconomic factors, including changes in diet, must underlie this change and would influence GCA through a spontaneous decrease of the $H$. pylori infection rate.

The present study had a number of limitations that should be taken into account before generalizing our findings. First, the patients in this study might not have been representative of the general population in Japan. However, because patients can seek medical care from any clinic or hospital in Japan, due to the national health insurance system, economic factors probably did not bias our findings. Second, the changes of $H$. pylori infection were not evaluated over the 25-year study period, but there are many previous reports regarding the decreased incidence of $H$. pylori infection in Japan $(20,21)$. Third, we did not evaluate drugs that 
can affect the GI tract, such as nonsteroidal antiinflammatory drugs (NSAIDs) or aspirin. There is a possibility that the range of indications for aspirin or NSAIDs is increasing as Japan's population ages. In this study, the incidence of patients with hematemesis or melena showed a gradual decrease over time-smaller than expected. This was possibly because peptic ulcer bleeding related to $H$. pylori has been decreasing, while bleeding related to drug consumption has conversely been increasing. Further studies will be necessary to assess differences in the trends of $H$. pylori-related and drug-related peptic ulcers. Fourth, we cannot exclude the possibility that there were some differences in the indication of upper GI endoscopy between the periods of 1981-1982, 1990-1991 and 2004-2005: in 1981-1982, barium examinations were often used as the screening test for upper GI diseases, such as peptic ulcer diseases or gastric cancers. An upper GI endoscopy was only performed when patients had abnormal findings in the barium examination. Therefore, the incidence of abnormal findings from upper GI endoscopies in 1981-1982 might be more frequent than those in 2004-2005. Further studies will be necessary to investigate the time trend of upper GI symptoms and final diagnosis using several tests including the barium examination.

In conclusion, in the future there will probably be fewer patients with GCA or peptic ulcers and more patients with GERD or dyspeptic patients with normal endoscopic findings among those with upper GI symptoms in Japan. Stratification of patients by a predictive model, so that endoscopy is only offered to those at high risk of organic dyspepsia, seems to be a reasonable approach for the future.

Abbreviations: GU: gastric ulcer, DU: duodenal ulcer, FD: functional dyspepsia, H. pylori: Helicobacter pylori, NSAIDs: nonsteroidal anti-inflammatory drugs

The authors state that they have no Conflict of Interest (COI).

\section{Acknowledgement}

We thank Ms. Makiko Kawakami for assistance of our work.

Data presented previously at DDW2007 and published as an abstract in Gastroenterology 2007; 132: A344.

\section{References}

1. Goh KL. Changing trends in gastrointestinal disease in the AsiaPacific region. J Dig Dis 8: 179-185, 2007.

2. El-Serag HB, Sonnenberg A. Opposing time trends of peptic ulcer and reflux disease. Gut 43: 327-333, 1998.

3. Wong SN, Sollano JD, Chan MM, et al. Changing trends in peptic ulcer prevalence in a tertiary care setting in the Phillippines: a seven-year study. J Gastroenterol Hepatol 20: 628-632, 2005.

4. Fujimoto K. Prevalence and epidemiology of gastro-oesophageal reflux disease in Japan. Aliment Pharmacol Ther 20(Suppl 8): 5-8, 2004.

5. Furukawa N, Iwakiri R, Koyama T, et al. Proportion of reflux esophagitis in 6010 Japanese adults: prospective evaluation by endoscopy. J Gastroenterol 34: 441-444, 1999.

6. Veldhuyzen van Zanten SJ, Flook N, Chiba N, et al. An evidence- based approach to the management of patients with dyspepsia in the era of Helicobacter pylori. CMAJ 162: S3-S23, 2000.

7. Talley NJ, Silverstein MD, Agreus L, Nyren O, Sonnenberg A, Holtmann G. AGA technical review: Evaluation of dyspepsia. American Gastroenterological Association. Gastroenterology 114: 582-595, 1998.

8. Talley NJ, Weaver AL, Zinsmeister AR. Impact of functional dyspepsia on quality of life. Dig Dis Sci 40: 584-589, 1995.

9. Drossman DA, Li Z, Andruzzi E, et al. U.S. householder survery of functional gastrointestinal disorders. Prevalence, sociodemography, and health impact. Dig Dis Sci 38: 1569-1580, 1993.

10. Heikkinen M, Pikkarainen $P$, Takala J, Räsänen H, Julkunen R. Etiology of dyspepsia: four hundred unselected consecutive patients in general practice. Scand J Gastroenterol 30: 519-523, 1995.

11. Kagevi I, Lofstedt S, Persson LG. Endoscopic findings and diagnoses in unselected dyspeptic patients at a primary health care center. Scand J Gastroenterol 24: 145-150, 1989.

12. Bytzer P, Hansen JM, Schaffalitzky de Muckadell OB. Over-thecounter availability of potent ulcer drugs. Study of changes in the drug use pattern and the pressure on diagnostic measures. Ugeskr Laeger 153: 1405-1410, 1991.

13. Moayyedi P, Zilles A, Clough M, Hemingbrough E, Chalmers DM, Axon AT. The effectiveness of screening and treating Helicobacter pylori in the management of dyspepsia. Eur J Gastroenterol Hepatol 11: 1245-1250, 1999.

14. Ladabaum U, Chey WD, Scheiman JM, Fendrick AM. Reappraisal of non-invasive management strategies for uninvestigated dyspepsia: cost-minimization analysis. Aliment Pharmacol Ther 16: 14911501, 2002.

15. Bowrey DJ, Griffin SM, Wayman J, Karat D, Hayes N, Raimes SA. Use of alarm symptoms to select dyspeptic for endoscopy causes patients with curable esophagogastric cancer to be overlooked. Surg Endosc 20: 1725-1728, 2006.

16. Barenys M, Abad A, Pons JM, et al. Scoring system has better discriminative value than Helicobacter pylori testing in patients with dyspepsia in a setting with high prevalence of infection. Eur J Gastroenterol Hepatol 12: 1275-1282, 2000.

17. Hassan C, Bersani G, Buri L, et al. Appropriateness of upper-GI endoscopy: an Italian survey on behalf of the Italian Society of Digestive Endoscopy. Gastrointest Endosc 65: 767-774, 2007.

18. van Kerkhoven LA, van Rijswijck SJ, van Rossum LG, et al. Is there any association between referral indications for open-access upper gastrointestinal endoscopy and endoscopic findings? Endoscopy 39: 502-506, 2007.

19. Mishima I, Adachi K, Arima N, et al. Prevalence of endoscopically negative and positive gastroesophageal reflux disease in the Japanese. Scand J Gastroenterol 40: 1005-1009, 2005.

20. Fujisawa T, Kumagai T, Akamatsu T, Kiyosawa K, Matsunaga Y. Changes in seroepidemiological pattern of Helicobacter pylori and hepatitis A virus over the last 20 years in Japan. Am J Gastroenterol 94: 2094-2099, 1999.

21. Haruma K, Okamoto S, Kawaguchi H, et al. Reduced incidence of Helicobacter pylori infection in young Japanese persons between the 1970s and the 1990s. J Clin Gastroenterol 25: 583-586, 1997.

22. Koike T, Ohara S, Sekine H, et al. Increased gastric acid secretion after Helicobacter pylori eradication may be a factor for developing reflux oesophagitis. Aliment Pharmacol Ther 15: 813-820, 2001.

23. Manabe N, Yoshihara M, Sasaki A, Tanaka S, Haruma K, Chayama K. Clinical characteristics and natural history of patients with low-grade reflux esophagitis. J Gastroenterol Hepatol 17: 949-954, 2002.

24. Ho KY, Chan YH, Kang JY. Increasing trend of reflux esophagitis and decreasing trend of Helicobacter pylori infection in patients from a multiethnic Asian country. Am J Gastroenterol 100: 1923- 
$1928,2005$.

25. Loffeld RJ. H. pylori and reflux esophagitis in Turkish patients living in the Zaanstreek region in the Netherlands. Dig Dis Sci 48: 1846-1849, 2003.

26. Hampel H, Abraham NS, El-Serag HB. Meta-analysis: obesity and risk for gastroesophageal reflux dosease and its complications. Ann Intern Med 143: 199-211, 2005.

27. Goh KL. Obesity and increasing gastroesophageal reflux disease in Asia. J Gastroenterol Hepatol 22: 1557-1558, 2007.

28. Nishikawa K, Sugiyama T, Kato M, et al. Non-Helicobacter pylori and non-NSAID peptic ulcer disease in the Japanese populations. Eur J Gastroenterol Hepatol 12: 635-640, 2000.

29. Ong TZ, Hawkey CJ, Ho KY. Nonsteroidal anti-inflammatory drug use is a significant cause of peptic ulcer disease in a tertiary hospital in Singapore: a prospective study. J Clin Gastroenterol 40: 795-800, 2006.

30. Haruma K, Sumii K, Uemura N, et al. Epidemiologic studies and recent changes of peptic ulcer disease during the past 10 years: study of 2548 cases collected during 1974 to 1983 in Hiroshima University Hospital. Hiroshima J Med Sci 35: 201-215, 1986.

31. Kawai K, Shirakawa K, Misaki F, Hayashi K, Watanabe Y. Natural history and epidemiologic studies of peptic ulcer disease in Japan. Gastroenterology 96: 581-585, 1989.

32. Goh KL, Wong HT, Lim CH, Rosaida MS. Time trends in peptic ulcer, erosive reflux oesophagitis, gastric and oesophageal cancers in a multiracial Asian population. Aliment Pharmacol Ther 29: 774-780, 2009.

\footnotetext{
(C) 2011 The Japanese Society of Internal Medicine http://www.naika.or.jp/imindex.html
} 